\title{
Surgical treatment of large hemangioma of the scrotum in a young adult male
}

\author{
Massimo Iafrate $^{1}$, Nicolò Leone ${ }^{1}$, Cesare Tiengo ${ }^{2}$, Filiberto Zattoni ${ }^{1}$ \\ ${ }^{1}$ Università degli studi di Padova, Dipartimento di Scienze Oncologiche Chirurgiche e Gastroenterologiche, Clinica Urologica, \\ Padova, Italy; \\ ${ }^{2}$ Università degli studi di Padova, Dipartimento di Neuroscienze, Chirurgia Plastica Ricostruttiva ed Estetica, Padova, Italy.
}

\begin{abstract}
Summary A 24-year-old male came to our clinic for a volumetric increase of a suspected scrotal hemangioma with thrombosis episodes. The ultrasound rose the suspicion of hemangioma and the Magnetic Resonance (MR) of the scrotum confirmed the suspicion. The mass was surgically removed and histopathology described a hemangioma cavernous. The postoperative course was regular and no subsequent relapse was shown in 5 months follow-up.
\end{abstract}

KEY WORDS: Andrology; Hemangioma; Scrotum; Scrotal surgery; Scrotal mass.

Submitted 3 February 2020; Accepted 29 February 2020

\section{INTRODUCTION}

Hemangiomas are the most common vascular neoplasms in children with an incidence in the first year of age ranging from 3 to $10 \%$. The most frequent localizations are head, neck, trunk, and extremities. Hemangiomas of the scrotum are rare conditions in adult. Many researchers consider them benign neoplasms originating from a congenital vascular anomaly (1).

\section{Case report}

After a written informed consent was obtained from the participant for the publication of this case report, we present a 24-year-old student male with a history of suspected scrotal hemangioma known from the age of 14 progressively growing over the past 12 months. In the previous 36 months, he went to the emergency room three times for hemangioma thrombosis which was conservatively treated. In medical history, he reported the removal of a lipoma in the sacral region and no previous traumas of genitalia. No significant family history was present. The physical examination showed a soft vascular mass of about $3 \times 7 \mathrm{~cm}$ positioned in the middle part of the scrotum (Figure 1). The testicles with their funiculum were completely independent from the vascular mass. The remaining physical examination did not show any other significant finding. Hematology, biochemistry, renal, liver function and hormonal panel were in range. MRI of the scrotum documented the presence of a lesion of $6 \times 7 \times 7 \mathrm{~cm}$ made of venous structures dilated developing in the context of the inferior-internal wall of the right scrotum containing some phlebolites, compatible with hemangioma. The lesion was in close contact with the right cavernous body but did not infiltrate Buck's fascia. Surgical excision was therefore planned. In general anesthesia, a diamond-shaped incision was made in the scrotal skin. With the dissection of the subcutaneous tissue, we identified the hemangioma inside the scrotum. After careful isolation of the neoplasm from the funicular structures and the testicles, we identified the site of origin at the perineal level on the Buck's fascia. The vessels were then tied to the site of origin with sutures, followed by complete removal of the hemangioma and sent to histological examination (Figure 2). A scrotal drainage in extraction and a bladder catheter were placed. Subcutaneous and skin have been sutured with detached points and a compressive dressing has been applied. The drainage and the catheter were removed the next day. The postoperative course was uneventful. Histopathological examination showed the presence of skin and subcutaneous tissue with vascular proliferation consisting of ectasic vessels with thin walls compatible with scrotal hemangioma. After 5 month, at the follow-up visit, the scar was nearly unrecognizable and there were no signs of recurrence (Figure 3).

\section{Discussion}

Cavernous hemangiomas of the scrotum are usually present since birth but come to the attention of the physician only during adolescence due to their unaesthetic aspect. Generally, they are painless masses but sometimes they can be associated with symptoms (pain and bleeding). The imaging helps to characterize the lesion, to assess the extent of the hemangioma, and to detect associated anomalies. Scrotal ultrasonography, however, is frequently not diriment for the diagnostic characterization. A hemangioma may be hypo or hyperechoic. The most typical finding is a mass of soft tissue containing phlebolites (small calcifications). The presence of phlebolites is characteristic of cavernous hemangioma. CT and MRI provide a simple and non-invasive method for the diagnosis and the determination of the extent of these lesions as well as their relationship with adjacent structures. For such reasons, these imaging techniques are considered mandatory before a surgical program (2). The therapeutic choice must take into account different aspects, from the size and the location of the neoplasm to the esthetic outcome. In the litera- 


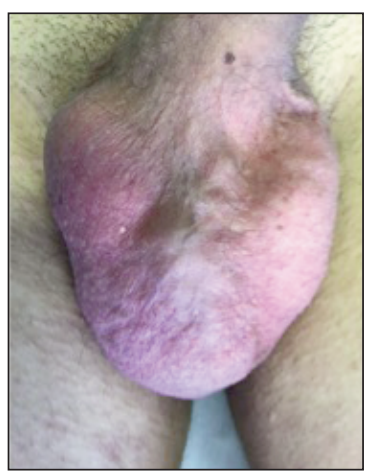

Figure 1.

Physical examination showed a soft vascular mass positioned in the middle part of the scrotum.

\section{Figure 2.}

Complete removal of the hemangioma for histological examination.
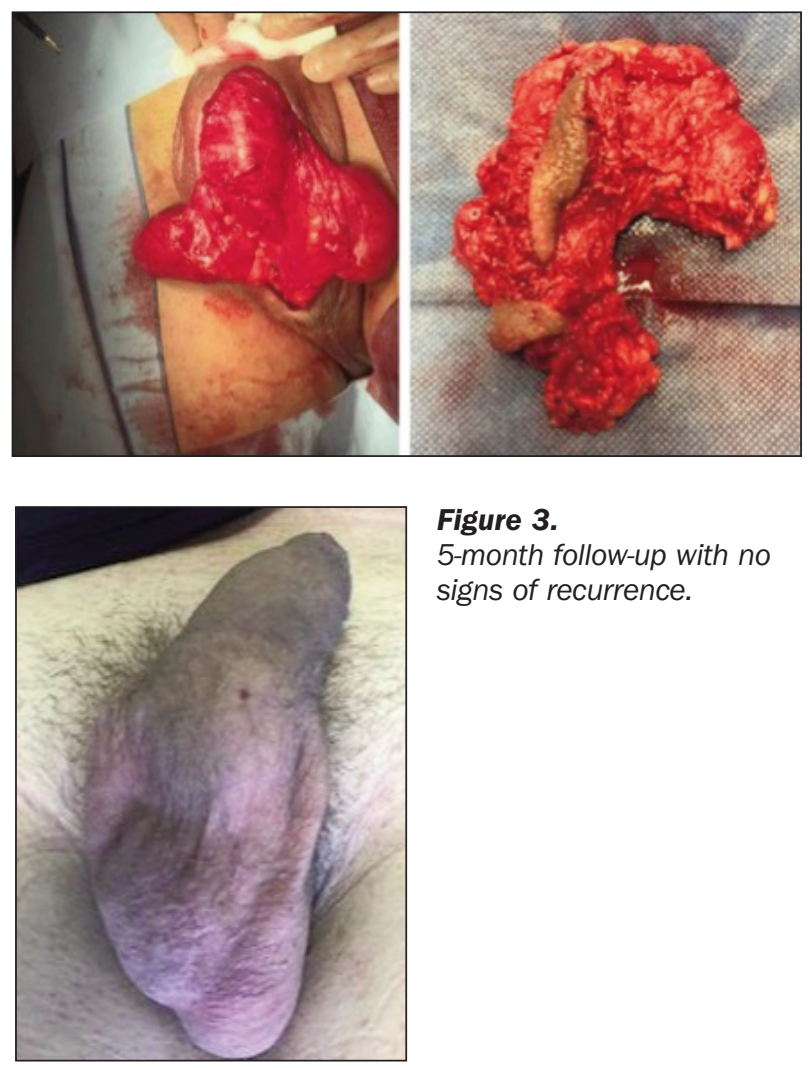

Figure 3.

5-month follow-up with no signs of recurrence. ture, good results are reported for small lesions both with $\mathrm{Nd}$ : YAG laser or carbon dioxide and with intralesional sclerotherapy. The surgical excision of these tumors of the genital area is burdened with the risk of bleeding due to the high vascularization and the possibility to develop unsightly scars (3). In this case, the surgical option was preferred since there were close relationships with the corpora cavernosa and we needed to avoid treatment options with high odd of necrosis of the erectile tissues.

\section{Conclusions}

The cavernous hemangioma of the scrotum is a rare entity and a diagnostic evaluation with MRI is mandatory in order to have a complete evaluation of the anatomic extension of the lesion. Surgical excision is a therapeutic choice with safe and satisfactory results, especially for large lesions in adulthood.

\section{References}

1. Lin CY, Sun GH, Yu DS, et al. Intrascrotal hemangioma. Arch Androl. 2002; 48:259-65.

2. Djouhri H, Arrivé L, Bouras T, et al. Diffuse cavernous hemangioma of the rectosigmoid colon: imaging findings. J Comput Assist Tomogr. 1998; 22:851-5.

3. Lee JM, Wang JH, Kim HS. Multiple cavernous hemangiomas of the glans penis, penis, and scrotum. Korean J Urol. 2008; 49:92-94.

\section{Correspondence}

Massimo Iafrate, MD (Corresponding Author) massimo.iafrate@unipd.it

Nicolò Leone, MD nicolo.leone@gmail.com

Filiberto Zattoni, MD

filiberto.zattoni@unipd.it

Clinica Urologica di Padova

Via Giustiniani 2 - 35100 Padova (Italy)

Cesare Tiengo, MD cesare.tiengo@unipd.it

Dipartimento di Neuroscienze, Chirurgia Plastica Ricostruttiva ed Estestetica, Padova (Italy) 